\title{
Evolução da Intervenção Percutânea para o Tratamento da Doença Coronária Multiarterial
}

\author{
Alexandre C. Zago, Amanda G.M.R. Sousa, José Eduardo Sousa \\ São Paulo, SP
}

A revascularização miocárdica em pacientes multiarteriais, ou seja, com lesão igual ou superior a 50\% em mais de uma artéria coronária, vem assumindo um crescente destaque no contexto da cardiologia intervencionista atual, já que, há alguns anos, era uma afecção eminentemente cirúrgica.

A angioplastia transluminal coronária com o balão foi investigada como opção terapêutica com resultados intrahospitalares satisfatórios, entretanto, a alta taxa de reestenose com conseqüente necessidade de reintervenção na evolução foram fatores limitantes desta técnica, principalmente em pacientes multiarteriais diabéticos.

O advento das endopróteses coronárias (stents) propiciou uma melhora importante dos níveis de reestenose em pacientes uniarteriais, o que motivou a utilização desta estratégia terapêutica também em multiarteriais. Os resultados dos estudos disponíveis (ERACI-II e ARTS) evidenciaram taxas semelhantes de eventos maiores irreversíveis (óbito, infarto e acidente vascular encefálico), quando comparados stents e cirurgia cardíaca, no período de um a três anos. Contudo, apesar da queda dos níveis de reestenose clínica pósimplante de stents em relação ao balão, ainda a cirurgia foi mais eficaz quanto à necessidade de reintervenção. Por outro lado, sabe-se que, idealmente, a melhor estratégia terapêutica é a que também oferece melhor custo-efetividade, e os stents comparados ao tratamento cirúrgico, no estudo ARTS, mostraram-se mais custo-efetivos.

No momento, frente às evidências, sugere-se a individualização do tratamento, analisando-se os aspectos clínicos e angiográficos; o risco pré, peri e pós-procedimento (conforme o risco de eventos cardiovasculares adversos maiores); e os custos. É igualmente necessário considerar a própria opinião do paciente após a exposição das possibilidades terapêuticas.

Instituto Dante Pazzanese de Cardiologia

Correspondência: Alexandre C. Zago - Serviço de Cardiologia Invasiva - Instituto Dante Pazzanese de Cardiologia - Av. Dr. Dante Pazzanese, 500 - 04012-180 São Paulo, SP - E-mail: azago@ rics.bwh.harvard.edu

Recebido para publicação em $1 / 2 / 01$

Aceito em 12/6/01
Destaque-se, contudo, que se torna necessária a constante realização de estudos comparativos, envolvendo os avanços recentes dos métodos de tratamento da doença arterial coronária para fundamentar a decisão da estratégia terapêutica, o mais adequadamente possível. Isto porque, particularmente, a intervenção percutânea é uma alternativa de revascularização em constante e rápida evolução, devido aos novos tipos de stents, às técnicas de otimização do implante dessas próteses e à terapêutica farmacológica antiplaquetária mais potente, que têm contribuído para a progressiva melhora dos resultados.

\section{Introdução}

O conceito doença coronária multiarterial pode ser definido como a presença de lesão igual ou superior a 50\%, pela análise visual da angiografia em duas ou mais projeções, em mais de uma artéria coronária ${ }^{1-5}$.

A importância do tema reflete-se na prática pelo aumento crescente de intervenções percutâneas em pacientes multiarteriais, que, no Brasil, passou de 34,4\% em 1992-93, para 38,8\% em 1996-97 do total de procedimentos terapêuticos realizados no país, correspondendo a um aumento de 2.894 casos em número absoluto, de acordo com os dados do registro nacional da Sociedade Brasileira de Cardiologia Intervencionista (CENIC) ${ }^{6}$, tendência também observada em outros países.

Há alguns anos, a doença coronária multiarterial era quase sinônimo de indicação de cirurgia de revascularização miocárdica, opção eficaz e superior aos resultados do tratamento clínico, conforme estudos randomizados das décadas de 70 e 80 demonstraram ${ }^{7-10}$. Entretanto, com o advento da angioplastia transluminal coronária, esta passou a ser uma opção a ser considerada ${ }^{11}$.

De fato, a intervenção coronária percutânea para multiarteriais apresenta uma série de vantagens, quando comparada à cirurgia cardíaca. Dentre elas, pode-se citar: o menor risco imediato de morbi-mortalidade cerebrovascular, pulmonar, digestiva e renal, principalmente em pacientes idosos e em portadores de doença prévia destes sistemas; 0 
tempo mais curto de convalescença e de internação hospitalar; o retorno precoce às atividades profissionais; e provavelmente, menores custos hospitalares e após a alta.

Tendo em perspectiva estes fatos, vários estudos randomizados foram desenhados nos anos 80 para estabelecer o méritorelativo entre as duas técnicas de revascularização em pacientes com doença em mais de um vasocoronárioprincipal.

\section{Estudos randomizados comparando angioplastia com o balão versus cirurgia}

Os principais estudos comparando o balão à cirurgia de revascularização miocárdica em pacientes multiarteriais são: RITA, ERACI, GABI, CABRI, EAST e BARI.

ORITA (Randomised Intervention Treatment of Angina Trial) teve como objetivo comparar os resultados clínicos de pacientes uni ou multiarteriais tratados com balão ou cirurgia de revascularização miocárdica, quando houvesse adequabilidade de ambas as formas de tratamento para o caso. Foram analisados 1.011 pacientes com doença em um vaso único ( $40 \%$ dos casos) ou em múltiplos (60\% dos pacientes) passíveis de ambas as formas de revascularização. Foram incluídos 555 pacientes com lesão $\geq 50 \%$ em mais de um vaso e angina estável ou instável. Foram excluídos os pacientes com doença em tronco de coronária esquerda, revascularização prévia, doença valvar hemodinamicamente significativa e doença não cardíaca com provável repercussão no prognóstico a longo prazo. Não houve diferença significante quanto à prevalência de óbito ou infarto agudo do miocárdio em 2,5 e 6,5 anos; entretanto, o grupo submetido à cirurgia de revascularização miocárdica apresentou menor taxa de angina e necessidade de intervenções diagnósticas e terapêuticas adicionais (tab. I) ${ }^{1,12}$.

O ERACI (Argentine Randomized Trial Coronary Angioplasty vs Bypass Surgery In Multivessel Disease) objetivou comparar os resultados a longo prazo de portadores somente de doença coronária multiarterial, submetidos à revascularização por cirurgia ou angioplastia com balão. Foram avaliados 127 pacientes com angina grave refratária ao tratamento medicamentoso, duas ou mais artérias coroná- rias principais com estenose $\geq 70 \%$ (análise visual) e possibilidade de revascularização completa por ambas as técnicas. Foram excluídos os pacientes com infarto agudo do miocárdio em evolução no momento da randomização, doença triarterial grave com fração de ejeção do ventrículo esquerdo $(\mathrm{FEVE}) \leq 35 \%$, estenose significativa em tronco de coronária esquerda, miocardiopatia dilatada isquêmica, miocardiopatia hipertrófica ou valvopatia importante, e doença não cardíaca que comprometesse a sobrevida. As complicações intra-hospitalares (óbito, infarto agudo do miocárdio e acidente vascular encefálico) foram similares em ambos os grupos. As taxas de óbito e infarto agudo do miocárdio também foram semelhantes em três anos; entretanto, a incidência de angina recorrente e a necessidade de revascularização adicional foram maiores no grupo balão (tab. I ${ }^{13}$.

O GABI ( German Angioplasty Bypass Surgery Investigation) teve como objetivo comparar a eficácia clínica entre angioplastia transluminal coronária com balão e cirurgia de revascularização miocárdica em pacientes com doença arterial coronária em dois ou três vasos, com a exigência de revascularização completa. Foram randomizados 359 pacientes, entre julho/86 e dezembro/91, com idades inferiores a 75 anos, angina classes II-IV (classificação da Canadian Cardiovascular Society), e estenose $\geq 70 \%$ nos vasos considerados para tratamento. Os critérios de exclusão foram: oclusão crônica; lesão em tronco de coronária esquerda (estenose $\geq 30 \%$ ); comprometimento estimado $\geq 50 \%$ do ventrículo esquerdo caso ocorresse oclusão abrupta de um dos vasos-alvo; lesões longas (>20mm); doença coronária periférica difusa; aneurisma do ventrículo esquerdo; infarto agudo do miocárdio nas quatro semanas prévias à randomização e revascularização prévia. Angioplastia e cirurgia resultaram em melhora semelhante da angina em um ano. $\mathrm{O}$ grupo submetido ao balão requereu, contudo, maior número de intervenções adicionais e fármacos antianginosos, enquanto o grupo submetido à cirurgia de revascularização miocárdica apresentou maior taxa de infarto agudo do miocárdio perioperatório (tab. I) ${ }^{14}$.

OCABRI ( Coronary Angioplasty versus Bypass Revascularization Investigation) teve como objetivo compa-

\begin{tabular}{|c|c|c|c|c|c|c|c|c|c|c|c|}
\hline & & & & bela I - & Iltados & studos co & balão & & & & \\
\hline \multirow[t]{2}{*}{ Estudo } & \multirow[t]{2}{*}{ Seguimento } & \multicolumn{2}{|c|}{ Mortalidade (\%) } & \multicolumn{2}{|c|}{$\operatorname{IAM}(\%)$} & \multicolumn{2}{|c|}{ Angina recorrente (\%) } & \multicolumn{2}{|c|}{$\operatorname{AVE}(\%)$} & \multicolumn{2}{|c|}{ Reintervenção (\%) } \\
\hline & & Balão & CRM & Balão & CRM & Balão & CRM & Balão & CRM & Balão & CRM \\
\hline \multirow[t]{2}{*}{ 1) RITA $^{1,12}$} & 2,5 anos & 3,1 & 3,6 & 6,7 & 5,2 & $31,3^{*}$ & $21,5^{*}$ & 1,8 & 2,0 & $37,1^{*}$ & $4,0^{*}$ \\
\hline & 6,5 anos & 7,6 & 9,0 & 10,8 & 7,4 & $30,9^{*}$ & $19,4^{*}$ & - & - & $53,0^{*}$ & $12,0^{*}$ \\
\hline \multirow[t]{2}{*}{ 2) $\mathrm{ERACI}^{13}$} & intra-hospitalar & 1,5 & 4,6 & 6,3 & 6,2 & - & - & 1,5 & 3,1 & - & - \\
\hline & 3 anos & 9,5 & 4,7 & 7,8 & 7,8 & $43^{*}$ & $21^{*}$ & - & - & $37^{*}$ & $6,3^{*}$ \\
\hline \multirow[t]{2}{*}{ 3) $\mathrm{GABI}^{14}$} & intra-hospitalar & 1,1 & 2,5 & $2,3^{*}$ & $8,1^{*}$ & $18^{*}$ & $7^{*}$ & 0 & 1,2 & - & - \\
\hline & 1 ano & 2,2 & 5,1 & 3,8 & 7,3 & 29 & 26 & - & - & $44^{*}$ & $6,0^{*}$ \\
\hline 4) $\mathrm{CABRI}^{2}$ & 1 ano & 3,9 & 2,7 & 4,9 & 3,5 & $13,9^{*}$ & $10,1^{*}$ & - & - & $33,6^{*}$ & $6,5^{*}$ \\
\hline \multirow[t]{2}{*}{ 5) EAST $^{15}$} & intra-hospitalar & 1,0 & 1,0 & $3,0^{*}$ & 10,3 & - & - & 0,5 & $1,5^{*}$ & - & - \\
\hline & 3 anos & 7,1 & 6,2 & 14,6 & 19,6 & - & - & - & - & $54^{*}$ & $13^{*}$ \\
\hline \multirow[t]{3}{*}{ 6) Bari ${ }^{4,16,17}$} & intra-hospitalar & 1,1 & 1,3 & $2,1^{*}$ & $4,6^{*}$ & - & - & 0,2 & 0,8 & - & - \\
\hline & 5 anos & 13,7 & 10,7 & 21,3 & 19,6 & - & - & - & - & $54,5^{*}$ & $8,0^{*}$ \\
\hline & 7 anos & $19,1^{*}$ & $15,6^{*}$ & - & - & - & - & - & - & $59,7^{*}$ & $13,1^{*}$ \\
\hline
\end{tabular}


rar os resultados clínicos (mortalidade, eventos cardíacos não-fatais e sintomas de angina) de pacientes multiarteriais submetidos ao balão ou à cirurgia de revascularização miocárdica como intervenção inicial para o tratamento de doença arterial coronária. Foram analisados 1.054 pacientes com idade inferior a 76 anos, angina estável ou instável e estenose $\geq 50 \%$ em dois ou mais vasos. Foram excluídos os pacientes com angioplastia transluminal coronária ou cirurgia de revascularização miocárdica prévias, estenose em tronco de coronária esquerda $\geq 50 \%$, vaso único pérvio, infarto agudo do miocárdio até dez dias antes da randomização, FEVE $\leq 35 \%$, insuficiência cardíaca, acidente vascular encefálico recente e doença grave que comprometesse a sobrevida. Uma particularidade desse estudo foi a inclusão de pacientes com vasos ocluídos e a não exigência de revascularização completa. O seguimento de um ano evidenciou risco equivalente de óbito em ambos os grupos; entretanto, o grupo balão necessitou maior número de reintervenções e fármacos antianginosos, assim como apresentou mais angina, principalmente em indivíduos do sexo feminino (tab. I $)^{2}$.

OEAST (Emory Angioplasty versus Surgery Trial) objetivou comparar os resultados do tratamento cirúrgico com os do balão em pacientes encaminhados para primeira revascularização coronária. Foram randomizados 392 casos, entre julho/87 e abril/90, com doença arterial coronária em dois ou três vasos, não submetidos à revascularização coronária prévia. Os critérios de exclusão foram: oclusão crônica (mais de oito semanas) com circulação colateral intracoronária; duas ou mais oclusões crônicas; lesão do tronco da coronária esquerda $\geq 30 \%$; FEVE $\leq 25 \%$; insuficiência cardíaca; infarto agudo do miocárdio até cinco dias antes da randomização; sintomas insuficientes para justificar a revascularização; e doença não cardíaca comprometendo a sobrevida. Não houve diferença entre angioplastia e cirurgia de revascularização miocárdica em relação ao objetivo composto primário, ou seja, mortalidade, IAM-Q e isquemia significativa evidenciada pela cintilografia miocárdica de esforço com tálio-201; porém, o grupo submetido à angioplastia com o balão apresentou maior necessidade de revascularização subseqüente em três anos (tab. I). Uma limitação desse estudo é o tamanho da casuística e o seguimento de três anos, que limitaram o poder estatístico e impediram uma avaliação rigorosa da mortalidade ${ }^{15}$.

O BARI (Bypass Angioplasty Revascularization Investigation) teve igualmente como objetivo comparar o balão e a cirurgia de revascularização miocárdica em pacientes com doença coronária multiarterial e angina ou isquemia grave, prevendo menor eficácia para intervenção percutânea. Esse foi o maior entre os estudos que compararam o balão e a revascularização cirúrgica. Foram analisados 1.829 pacientes, randomizados entre agosto/88 e agosto/91, com idades entre 18 e 79 anos e doença coronária multiarterial (lesão $\geq 50 \%$ em cada um dos vasos) com angina ou isquemia importante. Os critérios de exclusão foram: revascularização de emergência; lesão do tronco da coronária esquerda $\geq 50 \%$; doença não cardíaca que contra-indicasse angioplastia e cirurgia de revascularização miocárdica ou que li- mitasse a sobrevida; espasmo de coronária; calcificação importante na aorta ascendente; necessidade de cirurgia de grande porte ao mesmo tempo da revascularização e gestação conhecida ou suspeita. O grupo submetido à angioplastia com o balão apresentou taxas de sobrevida e IAM-Q semelhantes às do grupo submetido à cirurgia de revascularização miocárdica, em cinco anos; entretanto, os índices de revascularização subseqüentes foram significativamente maiores no grupo submetido ao balão. Nos pacientes diabéticos, a sobrevida em cinco anos foi significativamente maior no grupo tratado com cirurgia. Os resultados em sete anos mostraram maior sobrevida no grupo submetido à cirurgia de revascularização miocárdica, o que pode ser atribuído às diferenças de resultados no subgrupo dos diabéticos, pois, ao analisar-se separadamente o subgrupo dos diabéticos, verifica-se taxas de sobrevida mais elevadas com significância estatística nos pacientes submetidos à cirurgia de revascularização miocárdica, o que não ocorre quando se analisa isoladamente o subgrupo dos não-diabéticos. Quanto à necessidade de revascularização subseqüente em sete anos, os índices tiveram um aumento absoluto semelhante nos dois grupos durante o seguimento entre cinco e sete anos, portanto, a necessidade de revascularização subseqüente continuou significativamente maior no grupo submetido ao balão (tab. I) ) $^{3,4,16,17}$.

Uma análise dos fatores associados à mortalidade em cinco anos nos pacientes do estudo BARI evidenciou maior mortalidade cardíaca e geral nos pacientes diabéticos insulino-dependentes, submetidos à angioplastia com o balão, do que nos submetidos à cirurgia de revascularização miocárdica, com risco relativo de 2,63 e 1,78, respectivamente $(\mathrm{p}<0,001)$. Por outro lado, os pacientes com elevação ST submetidos à cirurgia de revascularização miocárdica apresentaram taxas mais elevadas de mortalidade cardíaca do que os submetidos ao balão, principalmente os acometidos por infarto agudo do miocárdio nas três semanas anteriores à revascularização miocárdica, com risco relativo de 4,08 $(\mathrm{p}<0,001)^{18}$.

O estudo Coronary Revascularization in Diabetic Patients, uma subanálise do estudo BARI, foi conduzido para investigar mais pormenorizadamente, nos resultados do estudo BARI, a evolução dos pacientes diabéticos. Esse estudo pesquisou a mortalidade, em cinco anos, de pacientes diabéticos submetidos ao balão ou à cirurgia de revascularização miocárdica, em dois subgrupos de pacientes: os diabéticos participantes do estudo BARI (353 pacientes) e os do BARI registro, ou seja, os elegíveis, porém não randomizados (339 pacientes), que foram submetidos às intervenções por decisão conjunta entre o médico e o paciente e que aceitaram participar de avaliações clínicas subseqüentes. Os critérios clínicos e angiográficos de inclusão e exclusão foram os mesmos delineados para o estudo BARI, entretanto, só foram analisados os pacientes com diabetes mellitus em tratamento com insulina ou hipoglicemiantes orais. Concluiu-se que os resultados dos pacientes registrados foram compatíveis com os achados dos pacientes randomizados, sendo que, também nestes, a cirurgia de revasculari- 


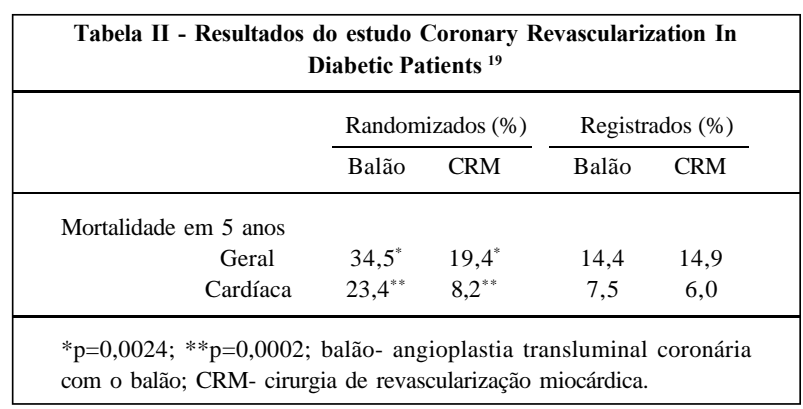

zação miocárdica esteve associada à maior sobrevida que a angioplastia transluminal coronária com o balão (tab. II). Esta subanálise possui limitações, uma vez que o estudo BARI não foi desenhado para avaliar pacientes diabéticos, pois não considerou nem a gravidade e a duração do diabetes mellitus, nem outros aspectos que pudessem influenciar os resultados. Outro fator importante foi o perfil mais favorável dos pacientes registrados, nos quais houve maior prevalência de raça branca, de atividade física e melhor qualidade de vida, além de nível de escolaridade mais alto, menor taxa de tabagismo e de insuficiência cardíaca, e menor prevalência de casos insulino-dependentes. Houve favorecimento angiográfico dos pacientes registrados submetidos ao balão, em relação aos submetidos à cirurgia de revascularização miocárdica, pois os primeiros apresentaram menor prevalência de doença triarterial, número de lesões significativas $\geq 4$, lesões ostiais e lesões na porção proximal da artéria descendente anterior esquerda. Essas limitações foram consideradas pelos autores ao analisar os resultados e elaborar a conclusão e não devem ser perdidas de vista ao considerarmos a revascularização nesses casos ${ }^{19}$.

A análise dos pacientes diabéticos do estudo BARI em sete anos evidenciou melhores resultados de sobrevida e necessidade de revascularização subseqüente no grupo submetido à cirurgia de revascularização miocárdica do que no grupo balão; entretanto, o efeito benéfico em relação à sobrevida somente foi demonstrado em pacientes revascularizados cirurgicamente com pelo menos uma artéria torácica interna, o que não foi condição para o efeito benéfico quanto à necessidade de reintervenção em sete anos. Outro resultado interessante desse estudo foi o aumento da necessidade de revascularização subseqüente, com significância estatística, em pacientes diabéticos submetidos ao

\begin{tabular}{|c|c|c|c|c|}
\hline \multicolumn{5}{|c|}{$\begin{array}{c}\text { Tabela III - Resultados do estudo Seven-Year Outcome in the } \\
\text { Bypass Angioplasty Revascularization Investigation (BARI) By } \\
\text { Treatment and Diabetic Status }{ }^{17}\end{array}$} \\
\hline & \multicolumn{2}{|c|}{$\mathrm{DM}(\%)$} & \multicolumn{2}{|c|}{ Não DM (\%) } \\
\hline & Balão & CRM & Balão & CRM \\
\hline Mortalidade em 7 anos & $44,3^{*}$ & $23,6^{*}$ & 13,2 & 13,6 \\
\hline Reintervenção em 7 anos & $69,9^{* *}$ & 11,1 & $57,8^{* *}$ & 13,5 \\
\hline \multicolumn{5}{|c|}{$\begin{array}{l}\text { * } \mathrm{p}=0,0011 ; * * \mathrm{p}=0,0078 ; \mathrm{DM} \text { - pacientes diabéticos; não DM- pacien- } \\
\text { tes não-diabéticos; balão: angioplastia transluminal coronária com o } \\
\text { balão; CRM- cirurgia de revascularização miocárdica. }\end{array}$} \\
\hline
\end{tabular}

balão quando comparados aos não-diabéticos, diferença que não foi constatada entre pacientes diabéticos e não-diabéticos submetidos à revascularização cirúrgica (tab. III) ${ }^{17}$.

Já o estudo Outcome of Coronary Bypass Surgery Versus Coronary Angioplasty in Diabetic Patients With Multivessel Coronary Artery Disease teve como objetivo comparar os resultados da cirurgia de revascularização miocárdica e da angioplastia transluminal coronariana com o balão em pacientes diabéticos com doença coronariana multiarterial, mediante a análise de uma base de dados observacional. Foram investigados 2.639 pacientes diabéticos, sem revascularização coronariana prévia, com doença em dois ou três vasos, submetidos à revascularização coronariana por angina estável ou instável ou por infarto agudo do miocárdio após vários dias de estabilização, entre janeiro/81 e dezembro/94. Os pacientes submetidos à revascularização coronariana de emergência por infarto agudo do miocárdio em evolução foram excluídos. Esse estudo evidenciou alta incidência de eventos fatais e não-fatais em pacientes diabéticos. Não houve diferença na sobrevida em 10 anos para diabéticos submetidos à cirurgia de revascularização miocárdica ou ao balão, assim como na análise específica dos diabéticos insulino-dependentes, apesar da menor gravidade do quadro clínico daqueles submetidos à intervenção percutânea e da maior prevalência de revascularização coronariana completa nos pacientes submetidos à cirurgia de revascularização miocárdica. Uma análise efetuada após a correção para as diferentes características dos grupos demonstrou maior mortalidade no grupo submetido ao balão, somente entre os pacientes insulino-dependentes. Houve maior incidência de procedimentos de revascularização coronariana adicionais no grupo submetido ao balão (tab. IV). A principal limitação desse estudo é a dificuldade de corrigir os vícios de seleção quanto à escolha do procedimento de revascularização, por se tratar de um estudo realizado com base de dados observacional ${ }^{20}$.

\section{Implante de próteses endocoronárias (stents)}

Até recentemente, os estudos disponíveis avaliando os resultados do implante de próteses coronárias (stents)

\begin{tabular}{|c|c|c|c|}
\hline \multicolumn{4}{|c|}{$\begin{array}{c}\text { Tabela IV - Resultados do estudo Outcome of Coronary Bypass } \\
\text { Surgery Versus Coronary Angioplasty in Diabetic Patients With } \\
\text { Multivessel Coronary Artery Disease }{ }^{20}\end{array}$} \\
\hline & Seguimento & Balão (\%) & $\operatorname{CRM}(\%)$ \\
\hline \multirow{3}{*}{ Mortalidade geral } & ntra-hospitalar & $0,36^{*}$ & $4,99^{*}$ \\
\hline & 5 anos & 22 & 24 \\
\hline & 10 anos & 55 & 52 \\
\hline \multicolumn{4}{|l|}{ Mortalidade } \\
\hline $\begin{array}{l}\text { (não corrigido } \\
\text { corrigido) }\end{array}$ & 10 anos & $69 / 64^{* *}$ & $62 / 53^{* *}$ \\
\hline \multirow{2}{*}{$\begin{array}{l}\text { Revascularização } \\
\text { adicional }\end{array}$} & 1 ano & $32^{*}$ & $1^{*}$ \\
\hline & 5 anos & $56^{*}$ & $8^{*}$ \\
\hline
\end{tabular}


em pacientes multiarteriais eram em sua maioria séries de casos, portanto, ao contrário dos estudos realizados com o balão já descritos, não havia, até meados dos anos 90, comparação simultânea com outras técnicas de revascularização, sendo as comparações efetuadas com os dados da literatura (controles históricos).

O estudo Clinical Outcome After Multivessel Coronary Stent Implantation teve como objetivo avaliar os resultados tardios de pacientes submetidos à intervenção coronária multiarterial com o implante destents. Foram avaliados 175 casos sem cirurgia de revascularização miocárdica prévia, submetidos à revascularização percutânea multiarterial com a proposta de implante de stents em todos os segmentos acometidos, entre janeiro/92 e março/98. O implante de stents nesses pacientes teve excelente taxa de sucesso $(98,3 \%)$, apesar da alta prevalência de lesões com características adversas. A sobrevida livre de eventos em um ano foi alta, sendo baixa a necessidade de reintervenção neste período (tab. V). As limitações desse estudo são: a análise retrospectiva, a casuística heterogênea, os múltiplos desenhos de stents, a variedade de estratégias de anticoagulação e a não uniformidade no uso de alta pressão para a expansão ótima do stent implantado ${ }^{21}$.

O estudoLong-term Angiographic and Clinical Outcome of Patients Undergoing Multivessel Coronary Stenting objetivou avaliar a segurança e a eficácia do implante de stents em multiarteriais e a possível redução da necessidade de reintervenções (particularmente da necessidade de cirurgia de revascularização miocárdica), já que os stents provaram ter um efeito anti-reestenótico. Foi analisada uma coorte de 100 pacientes multiarteriais consecutivos, submetidos ao implante de stents com alta pressão, entre março/93 e junho/95. Pacientes com lesões do tronco da coronária esquerda não protegido e FEVE <35\% foram excluídos. O im- plante de stents nesses pacientes multiarteriais foi realizado com alta taxa de sucesso (97\%) e baixa necessidade de cirurgia de revascularização miocárdica de emergência (2\%). Comparando com os resultados de estudos realizados com o balão, o implante de stents propiciou menor necessidade de reintervenção, em particular de cirurgia, e evidenciou taxa semelhante de sobrevida a longo prazo (tab. V). Entre as limitações desse estudo destacam-se a análise retrospectiva com os inerentes vícios de seleção para pacientes considerados apropriados para o implante de stents e os diferentes tipos de stents utilizados ${ }^{22}$.

O estudo Procedural Results and Late Clinical Outcomes Following Multivessel Coronary Stenting teve como meta avaliar os resultados intra-hospitalares e a longo prazo em uma grande série consecutiva de multiarteriais submetidos ao implante de stents. Foram então analisados 2.339 pacientes consecutivos, sendo 398 multiarteriais (dois ou três vasos) e 1.941 uniarteriais, tratados com o implante de stents entre janeiro/94 e agosto/97. Os pacientes submetidos à intervenção do tronco da coronária esquerda protegido ou não, bem como aqueles com procedimento estagiado, foram excluídos da análise. Os casos investigados haviam sido submetidos ao implante de stents em $67 \%$ das lesões-alvo, enquanto outros procedimentos de revascularização foram realizados nas demais lesões. Houve taxas semelhantes de sucesso e complicações maiores intra-hospitalares, assim como resultados clínicos similares a longo prazo, quando comparado o implante de stents em multiarteriais e uniarteriais (tab. V). Portanto, os autores concluíram que o implante de stents é uma estratégia terapêutica factível, eficaz e segura em pacientes com doença em mais de um vaso, cuidadosamente selecionados. Esse estudo apresenta limitações, como a análise retrospectiva, que determinou a exclusão de pacientes considerados melhores

\begin{tabular}{|c|c|c|c|c|c|c|}
\hline \multicolumn{7}{|c|}{ Tabela V - Resultados dos estudos com o implante de stents } \\
\hline Estudo & Seguimento & $\begin{array}{c}\text { Mortalidade } \\
(\%)\end{array}$ & $\begin{array}{l}\text { IAM } \\
(\%)\end{array}$ & $\begin{array}{c}\text { Angina Recorrente } \\
(\%)\end{array}$ & $\begin{array}{c}\text { Reintervenção } \\
(\%)\end{array}$ & $\begin{array}{l}\text { Evento (\%) } \\
\text { (óbito, IAM e } \\
\text { reintervenção) }\end{array}$ \\
\hline $\begin{array}{l}\text { Evolução clínica após } \\
\text { implante de múltiplos }\end{array}$ & Intra-hospitalar & 1,7 & 0 & - & - & - \\
\hline stents coronarianos 21 & 1 ano & 3,4 & 3,4 & 21 & 18,3 & 20,2 \\
\hline $\begin{array}{l}\text { Evolução clínica e } \\
\text { angiográfica dos } \\
\text { pacientes que } \\
\text { receberam múltiplos }\end{array}$ & Intra-hospitalar & 1 & $2(\mathrm{Q})$ & - & - & - \\
\hline stents coronarianos 22 & $21 \pm 10$ meses & 4 & - & $13^{+}$ & 30 & - \\
\hline $\begin{array}{l}\text { Resultado dos } \\
\text { poprocedimentos e }\end{array}$ & Intra-hospitalar & 0,5 & $0,9(\mathrm{Q})$ & - & - & - \\
\hline $\begin{array}{l}\text { evolução clínica tardia } \\
\text { após múltiplos implantes } \\
\text { de sents coronarianos }{ }^{23}\end{array}$ & 1 ano & 0,7 & $0(\mathrm{Q})$ & - & 21 & 22 \\
\hline $\begin{array}{l}\text { Resultados (>6 meses) } \\
\text { do implante de mais } \\
\text { de um stent em coronári }\end{array}$ & Intra-hospitalar & 0,7 & 4,4 & - & 0,7 & - \\
\hline $\begin{array}{l}\text { principal de doença } \\
\text { multiarterial coronariana }\end{array}$ & $18 \pm 13$ meses & 5,9 & 5,1 & - & 14,7 & $25^{\#}$ \\
\hline
\end{tabular}


candidatos à cirurgia de revascularização miocárdica (doença difusa triarterial, doença difusa envolvendo a artéria descendente anterior esquerda, lesões longas múltiplas, vasos de pequeno diâmetro, reestenose recidivante, reestenose intra-stent difusa e tentativa de revascularização percutânea prévia sem sucesso); a predominância de pacientes multiarteriais com doença em dois vasos; a maior taxa de reestudo nos pacientes uniarteriais, devido à sua inclusão em outros protocolos que envolviam reavaliação angiográfica, o que pode ter ocasionado aumento da detecção de reestenose e, conseqüentemente, aumento da taxa de reintervenção no grupo uniarterial; e a não inclusão de pacientes submetidos ao implante de stents em enxertos de veia safena ${ }^{23}$.

$\mathrm{O}$ estudo Results ( $>6$ Months) of Stenting of $>1 \mathrm{Ma}$ jor Coronary Artery in Multivessel Coronary Artery Disease avaliou 136 pacientes com o implante de stents em pelo menos duas artérias coronárias importantes, em uma ou duas etapas, com intervalo de tempo inferior a um mês entre as intervenções, no período de janeiro/95 a dezembro/97. Foram excluídos do estudo os pacientes com implante de stent em tronco de coronária esquerda e com história de cirurgia de revascularização miocárdica prévia. O perfil da casuística em estudo consistiu de doença coronária biarterial, função ventricular esquerda normal, baixa incidência de lesão em artéria descendente anterior e predomínio de lesão do tipo B (B2=55\%). A sobrevida livre de eventos cardíacos importantes - óbito, IAM-Q e não-Q e revascularização das lesões-alvo por intervenção percutânea ou cirurgia de revascularização miocárdica-foi de $75 \%$ em três anos. História de insuficiência cardíaca, intervenção em lesão reestenótica e intervenção em três vasos foram fatores prognósticos negativos independentes para sobrevida livre de eventos cardíacos maiores. A taxa de reestenose por lesão foi de $23 \%$ e, por paciente, de $36 \%$. Já o diâmetro de referência do vaso, o diâmetro luminal mínimo pós-intervenção e a extensão do stent foram fatores prognósticos independentes para reestenose. Os autores concluíram que o implante de stents em pacientes multiarteriais fornece bons resultados a médio prazo em pacientes selecionados (tab. V). Uma limitação importante desse estudo é a baixa taxa de reestudo angiográfico $(66 \%)$, que pode ter ocasionado uma taxa de reestenose superestimada, visto que a presença de sintomas e sinais de isquemia, provavelmente, contribuíram para incentivar a realização de reestudo angiográfico ${ }^{24}$.

O estudo ERACI II (Argentine Randomized Study Coronary Angioplasty With Stents Versus Coronary Bypass Surgery in Multiple Vessel Disease) teve como objetivo comparar os resultados do implante de stents com os da revascularização por cirurgia em pacientes multiarteriais nos períodos evolutivos de 30 dias, um, três e cinco anos. Foram avaliados 450 pacientes, sendo 225 submetidos à intervenção percutânea e 225 à cirurgia de revascularização miocárdica. No grupo angioplastia transluminal coronária, foram implantados 1,4 stents por paciente e utilizado abciximab em $28 \%$ dos casos. Os resultados em 30 dias evidenciaram menores taxas de mortalidade, IAM-Q e eventos cardíacos e cerebrovasculares adversos maiores (óbito, IAM-Q, aci- dente vascular encefálico e reintervenção) no grupo angioplastia com o implante destents, assim como menor período de internação hospitalar nesse mesmo grupo. Já a análise $18,5 \pm 6,4$ meses mostrou melhores resultados de sobrevidae IAM-Q no grupo angioplastia transluminal coronária, entretanto esse grupo apresentou taxa de angina recorrente mais elevada e maior necessidade de procedimentos adicionais de revascularização. Quanto aos custos, as duas técnicas mostraram-se similares na Argentina em 20 meses de evolução ${ }^{25-27}$ (tab. VI).

O estudo ARTS (Arterial Revascularization Therapy Study), o mais abrangente desta última série de investigações, objetivou comparar os resultados do implante de stents com a revascularização miocárdica por cirurgia em pacientes com doença coronária multiarterial. Foram avaliados 1.205 pacientes com angina estável ou instável ou com isquemia silenciosa, portadores de duas ou mais lesões $d e$ novo tratáveis com o implante de stents, em pelo menos duas artérias coronárias importantes. Esse estudo foi conduzido entre abril/97 e junho/98. Os critérios de exclusão foram: insuficiência cardíaca congestiva, necessidade de cirurgia de grande porte concomitante, infarto agudo do miocárdio transmural até sete dias prévios à randomização, história de acidente vascular encefálico e intenção de tratar mais de uma artéria coronária importante com oclusão crôni$\mathrm{ca}^{5,28}$. Os resultados evidenciaram uma redução de cerca de $50 \%$ na diferença de eventos cardíacos e cerebrovasculares adversos maiores - óbito, infarto agudo do miocárdio, acidente vascular encefálico e necessidade de reintervençãoem 12 meses entre as duas técnicas em estudo, com relação aos estudos anteriores. Ou seja, dos níveis de diferença de $30-40 \%$ nos estudos BARI, CABRI, EAST, GABI e RITA para $14 \%$ no estudo ARTS, ainda com resultados favoráveis à revascularização cirúrgica. A incidência dos três principais eventos clínicos irreversíveis -óbito, acidente vascular encefálico e infarto agudo do miocárdio-em 12 meses foi si-

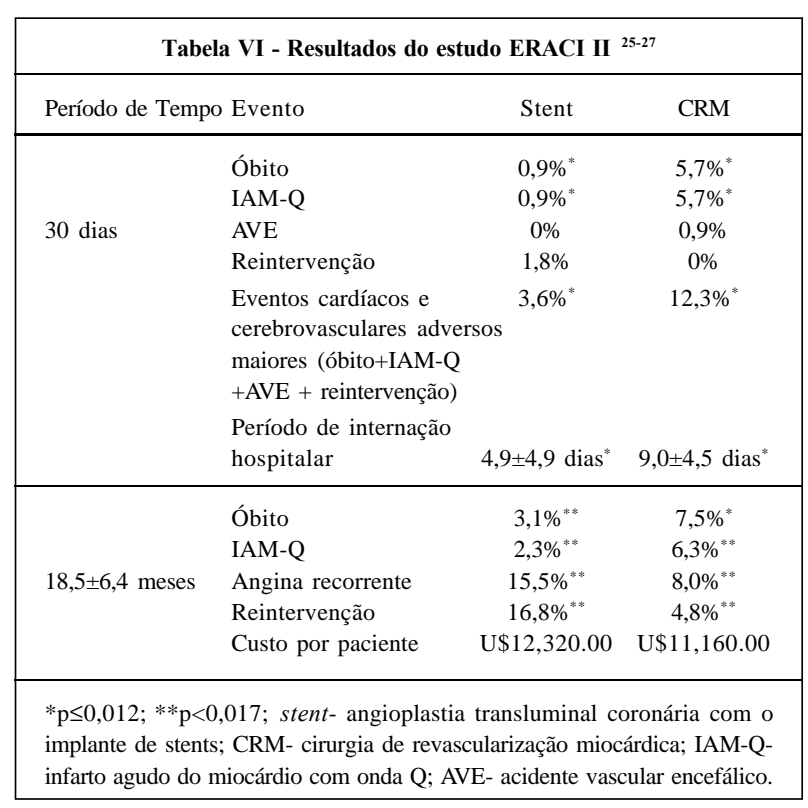


milar em ambas as técnicas de revascularização, assim como não foi diferente a sobrevida livre de eventos após a revascularização de pacientes bi ou triarteriais. A angina recorrente e a necessidade de reintervenção em 12 meses foram mais prevalentes no grupo submetido ao implante de stents, apesar do maior uso de nitratos e bloqueadores dos canais de cálcio nesse grupo. Os custos iniciais foram mais baixos no grupo submetido ao implante destents e permaneceram mais baixos em 12 meses, mesmo com uma certa queda da diferença inicial, devido aos custos correspondentes a ainda maior necessidade de revascularização adicional nesse grupo (tab. VII $)^{5}$.

Uma subanálise do estudo ARTS envolvendo 208 pacientes diabéticos, avaliou os resultados clínicos de pacientes diabéticos e não diabéticos submetidos à intervenção percutânea e à cirurgia. A mortalidade foi maior nos diabéticos submetidos à intervenção percutânea quando comparados aos não diabéticos; já a prevalência de acidente vascular encefálico foi maior nos diabéticos revascularizados por cirurgia quando comparados aos não diabéticos. A taxa de revascularização adicional foi maior nos não diabéticos submetidos à intervenção percutânea, quando comparados aos revascularizados por cirurgia (tab. VIII). Os índices de eventos cardiovasculares adversos maiores foram mais elevados em diabéticos submetidos à intervenção percutânea, quando comparados aos não diabéticos e em não diabéticos submetidos à intervenção percutânea, quando comparados aos revascularizados por cirurgia, sendo que a menor taxa de eventos maiores foi a dos não diabéticos tratados cirurgicamente (fig. 1) ${ }^{29}$.

Por fim, a subanálise Diabetic Patients With Multivessel Disease Treated With Percutaneous Coronary Revascularization Had Similar Outcome Than Those Treated With Surgery: One Year Follow Up Results From Two Argentine Randomized Studies (ERACI-ERACI II) objetivou avaliar os resultados tardios de pacientes diabéticos participantes de dois estudos randomizados: ERACI e ERACI II. Essas investigações que sortearam um total de 577 pacientes para tratamento com as duas técnicas de revascularização, percu-

\begin{tabular}{|c|c|c|}
\hline \multicolumn{3}{|c|}{ Tabela VII - Resultados do estudo ARTS em 12 meses $^{5}$} \\
\hline & Stent & CRM \\
\hline Óbito & $2,5 \%$ & $2,8 \%$ \\
\hline AVE & $1,5 \%$ & $2,0 \%$ \\
\hline IAM & $5,3 \%$ & $4,0 \%$ \\
\hline Óbito + AVE + IAM & $9,3 \%$ & $8,8 \%$ \\
\hline Angina recorrente & $21,1 \%{ }^{*}$ & $10,5 \% *$ \\
\hline Reintervenção & $16,8 \%^{*}$ & $3,5 \% \%^{*}$ \\
\hline \multicolumn{3}{|l|}{ Eventos cardíacos e } \\
\hline (óbito+AVE+IAM+reintervenção) & $26,2 \%^{*}$ & $12,2 \%^{*}$ \\
\hline procedimento & $\mathrm{U} \$ 6,441.00^{*}$ & U\$10,653.00* \\
\hline \multicolumn{3}{|l|}{ Custo por paciente } \\
\hline 1 ano & $\mathrm{U} \$ 10,665.00^{*}$ & $\mathrm{U} \$ 13,638.00^{*}$ \\
\hline \multicolumn{3}{|c|}{$\begin{array}{l}\text { *p }<0,001 ; \text { stent } \text { - angioplastia transluminal coronária com o implante de } \\
\text { stents; CRM- cirurgia de revascularização miocárdica; AVE- acidente } \\
\text { vascular encefálico; IAM- infarto agudo do miocárdio. }\end{array}$} \\
\hline
\end{tabular}

tânea e cirúrgica, incluíram na casuística 90 diabéticos, sendo 46 randomizados para cirurgia de revascularização miocárdica e 44 para angioplastia, entre os quais $86,4 \%$ dos casos (38/44) receberam stents. Não houve diferença estatisticamente significante entre ambos os grupos quanto à mortalidade, ao infarto agudo do miocárdio, à reintervenção e aos eventos cardiovasculares adversos maiores em um ano de evolução (tab. IX) ${ }^{30}$.

No Instituto Dante Pazzanese de Cardiologia, a análise dos 66 casos participantes do estudo ARTS, tratados no período de abril/97 a junho/98, evidenciou taxas semelhantes de óbito, infarto agudo do miocárdio e acidente vascular encefálico na fase hospitalar e em um ano. Entretanto, a necessidade de revascularização adicional foi maior no grupo submetido à intervenção percutânea após 12 meses de observação (tab. X). ${ }^{*}$

\section{Escolha da estratégia terapêutica}

A estratégia terapêutica de escolha é aquela que oferece os melhores resultados a curto e a longo prazo, com o menor risco de eventos cardíacos e cerebrovasculares adversos importantes, associada a uma relação custo/benefício satisfatória.

Os estudos de intervenção percutânea com o balão em multiarteriais evidenciaram sucesso em $87-92 \%$ dos casos; mortalidade em 1,0-1,5\%; infarto agudo do miocárdio em 2,16,3\%; e cirurgia de revascularização miocárdica de emergência em 1,5-10,3\% no período hospitalar ${ }^{1,2,4,12-17,31}$. As taxas de mortalidade e acidente vascular encefálico foram semelhantes entre os grupos balão e cirurgia de revascularização miocárdica; entretanto, os pacientes submetidos à intervenção percutânea apresentaram menor taxa de infarto agudo do miocárdio e tempo de internação hospitalar mais curto $^{31}$. Analisando-se os resultados do desempenho do balão a longo prazo, ou seja, com um a sete anos de evolução, a mortalidade foi de 2,2-19,1\%; o infarto agudo do miocárdio ocorreu em 3,8-21,3\%; e a reintervenção foi necessária em 33,6-59,7\%. Não houve diferença significante quanto à mortalidade e ao infarto agudo do miocárdio entre as duas formas de tratamento, porém, os pacientes tratados com o balão necessitaram maior número de novos procedimentos de revascularização ${ }^{1,2,4,12-17}$.

Esses dados permitem-nos concluir que a intervenção percutânea com o balão, ainda que apresente bons resultados iniciais com baixa taxa de complicações e curto período de convalescença, proporcionando grande conforto ao paciente, não favorece a evolução tardia, pois os resultados a longo prazo não são satisfatórios o suficiente, devido à alta incidência de angina recorrente com necessidade de reintervenção. Ainda assim, acompanha-se de baixa taxa de eventos cardíacos adversos maiores, como mortalidade e infarto agudo do miocárdio, comparáveis aos resultados da revascularização cirúrgica.

"Sousa JE. Comunicação Pessoal ao $55^{\circ}$ Congresso Brasileiro de Cardiologia, Recife - PE. 


\begin{tabular}{|c|c|c|c|c|}
\hline \multicolumn{5}{|c|}{ Tabela VIII - Resultados da subanálise de pacientes diabéticos do estudo ARTS em um ano ${ }^{29}$} \\
\hline Eventos & $\mathrm{DM}+/$ stent $(\mathrm{n}=112)$ & DM-/stent $(\mathrm{n}=488)$ & $\mathrm{DM}+/ \mathrm{CRM}(\mathrm{n}=96)$ & DM-/CRM (n=508) \\
\hline Óbito & $6,3 \%^{*}$ & $1,6 \%{ }^{*}$ & $3,1 \%$ & $2,4 \%$ \\
\hline AVE & $2,7 \%$ & $1,6 \%$ & $6,3 \%{ }^{* *}$ & $1,4 \%{ }^{* *}$ \\
\hline IAM & $8,9 \%$ & $5,5 \%$ & $3,1 \%$ & $3,9 \%$ \\
\hline CRM & $9,8 \%$ & $5,9 \%$ *** & $2,1 \%$ & $0,6 \%$ *** \\
\hline ATC & $21,4 \%$ & $15,0 \% \%^{* * *}$ & $2,1 \%$ & $3,5 \%^{* * *}$ \\
\hline $\begin{array}{l}\text { *DM+/ste } \\
\text { não-diabé } \\
\text { encefálico }\end{array}$ & $\begin{array}{l}\text { stent }(\mathrm{p} \leq 0,011) ; * * \mathrm{D} \\
\text { angioplastia translu } \\
\text { ito agudo do miocár }\end{array}$ & $\begin{array}{l}\text { M-/CRM ( } \leq 0,01) \\
\text { a com o implante de }\end{array}$ & $\begin{array}{l}\text { DM-/CRM ( } \mathrm{p}<0,01) ; \\
\text { irurgia de revasculari }\end{array}$ & $\begin{array}{l}\text { diabéticos; DM-: pacier } \\
\text { a; AVE- acidente vasc }\end{array}$ \\
\hline
\end{tabular}

\begin{tabular}{|c|c|c|}
\hline \multicolumn{3}{|c|}{$\begin{array}{l}\text { Tabela IX - Resultados da análise de pacientes diabéticos } \\
\text { multiarteriais dos estudos ERACI e ERACI II em um ano }{ }^{30}\end{array}$} \\
\hline & $\operatorname{ATC}(\%)$ & CRM $(\%)$ \\
\hline Óbito & 4,5 & 6,5 \\
\hline IAM & 0 & 6,5 \\
\hline Reintervenção & 19,0 & 7,0 \\
\hline $\begin{array}{l}\text { Eventos cardiovasculares } \\
\text { adversos maiores }\end{array}$ & & \\
\hline (óbito+IAM+reintervenção) & 22,7 & 19,5 \\
\hline
\end{tabular}

\begin{tabular}{|c|c|c|}
\hline \multicolumn{3}{|c|}{$\begin{array}{l}\text { Tabela X - Resultados da análise de pacientes multiarteriais do } \\
\text { estudo ARTS tratados no Instituto Dante Pazzanese de Cardiologia }\end{array}$} \\
\hline Idade & $\begin{array}{l}\text { Stent }(\mathrm{n}=33) \\
54,9 \text { anos }\end{array}$ & $\begin{array}{l}\text { CRM (n=33) } \\
55,2 \text { anos }\end{array}$ \\
\hline Sexo masculino & $76 \%$ & $70 \%$ \\
\hline Diabete melito & $18 \%$ & $24 \%$ \\
\hline Angina instável & $36 \%$ & $31 \%$ \\
\hline \multicolumn{3}{|l|}{ Eventos hospitalares } \\
\hline IAM & $3 \%$ & $3 \%$ \\
\hline CRM de emergência & $0 \%$ & $0 \%$ \\
\hline AVE & $0 \%$ & $3 \%$ \\
\hline Óbito & $0 \%$ & $0 \%$ \\
\hline \multicolumn{3}{|l|}{ Eventos em 1 ano } \\
\hline IAM & $3 \%$ & $3 \%$ \\
\hline AVE & $0 \%$ & $3 \%$ \\
\hline Óbito & $0 \%$ & $0 \%$ \\
\hline $\begin{array}{l}\text { Revascularização adicional } \\
\text { (CRM/ATC) }\end{array}$ & $12 \% \%^{*} / 15 \% * *$ & $0 \%{ }^{*} / 3 \%{ }^{* *}$ \\
\hline $\begin{array}{l}\text { Sobrevida livre de eventos } \\
\text { (óbito+IAM+AVE+reintervenção) }\end{array}$ & $70 \%{ }^{* * *}$ & $91 \%^{* * * *}$ \\
\hline \multicolumn{3}{|c|}{$\begin{array}{l}\text { \# Comunicação pessoal de Sousa JE ao } 55^{\circ} \text { Congresso da Sociedade } \\
\text { Brasileira de Cardiologia, Recife - PE. } \\
\text { *p=0,04; **p }=0,08 ; * * * \mathrm{p}=0,03 ; \text { stent- angioplastia transluminal } \\
\text { coronária com o implante de stents; CRM- cirurgia de revascularização } \\
\text { miocárdica; IAM- infarto agudo do miocárdio; AVE- acidente vascular } \\
\text { encefálico; ATC- angioplastia transluminal coronária. }\end{array}$} \\
\hline
\end{tabular}

Os estudos analisados, entretanto, apresentam algumas limitações e divergências relevantes, que devem ser destacadas. Somente uma pequena porcentagem de pacientes com doença coronária multiarterial preencheu os rígidos critérios de inclusão e uma porcentagem ainda inferior foi de fato randomizada (5 a 10\%). Quanto ao quadro clínico de admissão, observou-se que as taxas de infarto agudo do miocárdio foram diferentes entre os estudos, o que pode ser atribuído às divergências quanto aos critérios de diagnóstico e classificação do infarto agudo do miocárdio. Assim, enquanto o estudo EAST incluiu infarto agudo do miocárdio silencioso diagnosticado pela presença de novas ondas Q no eletrocardiograma, outros estudos incluíram somente infarto agudo do miocárdio diagnosticado clinicamente ${ }^{32}$. Houve ainda significativa variação dos fatores de risco nas casuísticas analisadas. Pacientes com oclusões crônicas foram excluídos de alguns estudos, mas não de outros ${ }^{31}$. Além disto, a maioria dos pacientes randomizados era biarterial e possuía função ventricular esquerda normal, o que levou à seleção de coronarianos com evolução provavelmente mais favorável. Todos esses aspectos clínicos e angiográficos demonstram que o universo de multiarteriais investigado foi relativamente restrito, relacionado a pacientes de moderada complexidade clínica e angiográfica. O critério de revascularização completa, anatômica ou funcional, o uso de novos dispositivos de revascularização e a utilização da artéria torácica interna esquerda ou direita na revascularização cirúrgica também variaram entre os estudos. De grande importância são os critérios para revascularização adicional, que também foram diferentes, pois enquanto os estudos RITA, ERACIe BARI estabeleceram critérios clínicos para a indicação de nova cineangiocoronariografia, os estudos CABRI,EASTe GABI determinaram a realização de cineangiocoronariografia adicional de rotina, o que pode ter ocasionado uma taxa superestimada de reintervenção nos estudos que incluíram reestudo angiográfico rotineiro.

$\mathrm{O}$ advento dos stents representou um importante avanço no tratamento percutâneo da doença arterial coronária, pois propiciou uma significativa melhora dos resultados angiográficos imediatos, assim como diminuição das taxas de oclusão aguda com acentuado efeito anti-reestenótico. Os estudos Benestent I, Benestent II e Stress I em coronarianos portadores de doença uniarterial evidenciaram taxas de reestenose de $32 \%, 31 \%$ e $42,1 \%$ para os pacientes submetidos ao balão, enquanto de $22 \%, 16 \%$ e $31,6 \%$ para aqueles tratados com o implante destents. Analisandose a incidência de eventos cardiovasculares adversos maiores em seis meses, esses estudos apresentaram taxas de $29,6 \%, 19,3 \%$ e $23,8 \%$ para os submetidos ao balão e de $20,1 \%, 12,8 \%$ e $19,5 \%$ para os tratados com o implante de stents $^{33-35}$. Baseando-se na significativa melhora dos resultados nesses pacientes uniarteriais, decidiu-se comparar os 
resultados do implante de stents com os da revascularização cirúrgica em multiarteriais.

As séries iniciais com o emprego dos stents nos pacientes multiarteriais mostraram taxas favoráveis de mortalidade $(0,7-5,9 \%)$, de reintervenção $(14,7-30 \%)$ e de eventos cardiovasculares adversos $(20,2-25 \%)$ a longo prazo ${ }^{21-24}$, melhores dos que as obtidas com o balão em multiarteriais disponíveis na literatura. Com os stents, portanto, a intervenção percutânea tornava-se mais competitiva em relação à cirurgia. Surgia então a necessidade de repetir-se as investigações passadas, agora com o uso prioritário dos stents na revascularização percutânea e o emprego de enxertos arteriais na revascularização cirúrgica. Deste modo, os estudos ARTS e ERACIII foram realizados para avaliar esta questão, utilizando stents de segunda e terceira geração, assim como protocolo farmacológico anti-agregante plaquetário atual. Essas investigações confirmaram os bons resultados iniciais que o implante de stents havia apresentado em multiarteriais, ou seja, mortalidade de 2,5 e 3,1\% em 12 e 20 meses, respectivamente, e necessidade de revascularização adicional de $16,8 \%$ em ambos os estudos ${ }^{5,27}$. O estudo ARTS também evidenciou uma taxa de eventos cardiovasculares adversos maiores de $26,2 \%$ e ao se comparar os resultados do implante de stents com os da revascularização cirúrgica, verificaram-se resultados similares em relação aos principais eventos clínicos irreversíveis (óbito, acidente vascular encefálico e infarto agudo do miocárdio). Entretanto, a angina recorrente ainda foi pouco mais prevalente no grupo submetido aos stents ( $21 \%$ versus $11 \%$ ), atingindo a diferença significância estatística ${ }^{5}$.

Apesar da indiscutível importância dos resultados dos estudos ARTS e ERACI II, outras investigações no mesmo sentido estão sendo conduzidas para o esclarecimento definitivo da melhor estratégia terapêutica no grande universo dos multiarteriais, envolvendo outros tipos de stents e diferentes situações clínicas e angiográficas.

No momento, sugere-se a individualização da estratégia terapêutica, analisando-se os riscos pré, peri e pós-procedimento, os de reestenose e de eventos cardiovasculares adversos maiores na evolução, além da questão não menos importante da custo-efetividade. Considerar-se a opinião do paciente após a exposição das opções terapêuticas também deve ser meta importante na tomada de decisão. Convém ressaltar, principalmente no caso de pacientes jovens, e sendo a doença progressiva, a possibilidade de reintervenções ao longo dos anos. Os procedimentos cirúrgicos repetidos apresentam riscos mais elevados de morbi-mortalidade e, em muitas situações, menor taxa de sucesso no alívio de sintomas, o que nos leva a refletir sobre a preferência da estratégia percutânea com o implante de stents como tratamento inicial, sempre que possível, com o intuito de evitar ou de postergar a revascularização cirúrgica, eliminando ou reduzindo as cirurgias, na história natural desses casos.

Os pacientes diabéticos, que compreendem 20-30\% dos casos de coronariopatia tratada com revascularização miocárdica percutânea ou cirúrgica, requerem uma reflexão particular. O diabetes mellitus é um importante fator de risco para o desenvolvimento de doença arterial coronária e constitui um fator prognóstico independente de evolução adversa a longo prazo, pois a hiperglicemia e a hiperinsulinemia aceleram o desenvolvimento e a progressão da coronariopatia, devido à disfunção endotelial e ao aumento da ativação plaquetária, da atividade pró-trombótica e da produção de fatores de crescimento ${ }^{36}$.

São escassos os dados disponíveis comparando as diferentes intervenções em pacientes diabéticos multiarteriais; entretanto, observa-se que a mortalidade a longo prazo é maior, de um modo geral, nos pacientes submetidos à angioplastia, quando comparados aos tratados cirurgicamente, ainda que qualquer das formas de revascularização acarrete mortalidade mais elevada nos diabéticos do que na população geral. Quanto à necessidade de revascularização miocárdica adicional, devido à maior propensão para a reestenose, os diabéticos também mostram taxas mais elevadas de reintervenção nos casos tratados inicialmente por via percutânea. Parece que o uso dos stents associados aos inibidores da GP IIb/IIIa pode mudar este prognóstico nos uniarteriais e, talvez, também nos portadores de doença de mais de um vaso ${ }^{37}$.

Não há estudos randomizados prospectivos avaliando os resultados do implante de stents em pacientes multiarteriais diabéticos, entretanto o estudo Procedural Results and Late Clinical Outcomes Following Multivessel Coronary Stenting evidenciou novamente o diabetes melito como fator prognóstico negativo independente de eventos cardíacos (revascularização da lesão-alvo e qualquer revascularização repetida) ${ }^{23}$.

A subanálise já mencionada dos estudos ERACI e ERACI II em diabéticos multiarteriais não verificou diferença estatisticamente significante entre implante de stents e cirurgia de revascularização miocárdica quanto à mortalidade, ao infarto agudo do miocárdio, as reintervenções e aos eventos cardiovasculares adversos maiores ${ }^{30}$. Já a subanálise dos diabéticos do estudo ARTS demonstrou resultados clínicos desfavoráveis da intervenção percutânea com os stents quando comparada à cirurgia, apesar do risco significativo de acidente vascular encefálico dos diabéticos submetidos à revascularização cirúrgica ${ }^{29}$.

Portanto, torna-se pertinente a realização de estudos randomizados prospectivos que avaliem a difícil questão do diabetes melito, especialmente agora quando os resultados dos stents podem ser ainda melhorados com a adição de fármacos antiagregantes plaquetários ou com os stents recobertos com substâncias antiproliferativas.

A julgar pelos achados do estudo EPISTENT ( $\mathrm{Com}$ plementary Clinical Benefits of Coronary-Artery Stenting and Blockade of Platelet Glycoprotein IIb/IIIa Receptors) é possível que os inibidores da GP IIb/IIIa, em particular o abciximab (que possui reação cruzada com o receptor para a vitronectina), possam ter um impacto favorável adicional ao dos stents nos resultados tardios, pois a associação de abciximab e stents em pacientes diabéticos evidenciou uma menor taxa de reintervenção em seis meses $(8,1 \%)$ quando comparada aos diabéticos submetidos a stent e placebo 
(16,6\%) ou balão e abciximab (18,4\%), com significância estatística, assim como esta taxa foi similar à dos não-diabéticos submetidos a stent e abciximab $(8,7 \%)^{37}$.

Além disto, a perspectiva do uso de stents recobertos com rapamicina (fármaco antiproliferativo), que resultaram em uma taxa de reestenose e eventos cardiovasculares adversos maiores (óbito, infarto agudo do miocárdio, trombose intra-stent e reintervenção) de 0\% em doze meses, em um estudo realizado no Instituto Dante Pazzanese de Cardiologia, no qual $20 \%$ dos pacientes eram diabéticos, representa uma estratégia possivelmente útil para aumentar a eficácia tardia da endoprótese, com particular interesse neste subgrupo de pacientes, sabidamente mais passíveis de reestenose ${ }^{38}$.

Outra estratégia que vem apresentando bons resultados é a braquiterapia. Os estudos WRIST (Intracoronary g-Radiation Therapy After Angioplasty Inhibits Recurrence in Patients With In-Stent Restenosis) e Gamma-One (Localized Intracoronary Gamma-Radiation Therapy to Inhibit the Recurrence of Restenosis After Stenting) avaliaram os resultados da radiação gama em lesões reestenóticas, evidenciando uma taxa de reestenose em seis meses de $22 \%$ e $32 \%$ versus $60 \%$ e $55 \%$ no grupo placebo, respectivamente $(\mathrm{p}=0,0001 \mathrm{e} 0,01)^{39,40}$.

Já o estudo PREVENT (Inhibition of Restenosis With b-Emitting Radiotherapy) comparou a radiação beta com o placebo em lesões de novo (70\%) e reestenóticas (30\%), com taxa de reestenose em seis meses de $22 \%$ versus $50 \%$ $(\mathrm{p}=0,018)$, enquanto o estudo Beta WRIST (Intracoronary b-Radiation Therapy Inhibits Recurrence of In-Stent Restenosis) analisou os resultados da radiação beta somente em lesões reestenóticas com taxa de reestenose em seis meses de $34 \%$ versus $71 \%$ no grupo placebo $(\mathrm{p}=0,001)^{41,42}$.

O estudo SCRIPPS (Catheter-Based Radiotherapy to Inhibit Restenosis After Coronary Stenting) comparou o uso da radiação gama com o placebo em lesões reestenóticas, mostrando uma taxa de reestenose de $17 \%$ versus $54 \%$ em seis meses $(\mathrm{p}=0,01)$ e de $33 \%$ versus $64 \%$ em três anos $(\mathrm{p}<0,05)^{43,44}$. Portanto, apesar da radiação gama ocasionar uma redução de $69 \%$ na taxa de reestenose em seis meses e esta diminuir para $48 \%$ em três anos, ainda assim a redução da taxa de reestenose em três anos permaneceu significativa, o que demonstra os efeitos benéficos da braquiterapia a longo prazo. Contudo, deve-se aguardar os resultados tardios dos estudos com radiação beta e gama em andamento para o melhor esclarecimento dos resultados a longo prazo da braquiterapia. Outra questão igualmente importante é a trombose tardia, ocorrida em 5,3-10,0\% dos pacientes tratados com radiação ${ }^{39-42}$, especialmente após a interrupção da ticlopidina ou do clopidogrel, o que indica a necessidade de se reavaliar a duração do tratamento com antiagregantes plaquetários nos pacientes submetidos a braquiterapia.

De qualquer forma, na problemática evolução dos diabéticos, dois aspectos devem sempre estar na mente dos clínicos. Primeiramente, a perspectiva das formas inusitadas de apresentação clínica da reestenose que, freqüentemente, é silenciosa ou surge como arritmias fatais nos diabéticos. Daí a necessidade de aumentar a periodicidade e a indicação dos testes detectores de isquemia. Além disso, a doença exige cuidados clínicos rigorosos (controle dos níveis glicêmicos e dos fatores de risco cardiovasculares), que podem potencializar os bons resultados de qualquer das opções de revascularização.

Em conclusão, a intervenção percutânea tem apresentado resultados imediatos e tardios cada vez mais eficazes com o desenvolvimento de novos tipos de stents, de técnicas de otimização de implante e da terapêutica farmacológica associada, contribuindo para a melhora dos resultados tanto em uni quanto em multiarteriais.

Apesar dos avanços na área cirúrgica - a introdução da proteção miocárdica, a crescente utilização de enxertos arteriais e os procedimentos minimamente invasivos e/ou conjugados (associação de intervenções percutâneas e cirúrgicas), que também aprimoraram a evolução pós-operatória dos multiarteriais - a magnitude da diferença dos resultados entre cirurgia e angioplastia vem progressivamente sendo reduzida, às custas do expressivo desenvolvimento da cardiologia intervencionista.

A terapêutica farmacológica associada e os stents recobertos com fármacos antiproliferativos, principalmente a rapamicina, ${ }^{45}$ têm apresentado resultados promissores, especialmente na redução da oclusão (sub) aguda, da reestenose e, conseqüentemente, da revascularização adicional.

Atualmente, sugere-se a individualização da estratégia terapêutica, analisando-se os riscos pré, peri e pós-procedimento, os de reestenose e os de eventos cardiovasculares adversos maiores na evolução, assim como considerar a custo-efetividade e a opinião do paciente após a exposição das opções terapêuticas.

\section{Referências}

1. RITA trial participants. Coronary angioplasty versus coronary artery bypass surgery: the Randomised Intervention Treatment of Angina (RITA) trial. Lancet 1993; 341: 573-80.

2. CABRI Trial Participants. First-year results of CABRI (Coronary Angioplasty versus Bypass Revascularisation Investigation). Lancet 1995; 346: 1179-84.

3. Protocol for the Bypass Angioplasty Revascularization Investigation. Circulation 1991; 84(suppl V): V-1-V-27.

4. The Bypass Angioplasty Revascularization Investigation (BARI) Investiga- tors. Comparison of coronary bypass surgery with angioplasty in patients with multivessel disease. N Engl J Med 1996; 335: 217-25.

5. Serruys PW, Unger F, Sousa JE, et al. Comparison of coronary-artery bypass surgery and stenting for the treatment of multivessel disease. N Engl J Med 2001; 344: 1117-24

6. Sousa JE, Sousa AG, Mattos LA, Pinto I. Coronary angioplasty in Brazil. Rev Port Cardiol 1999; 18 Suppl I: I31-5.

7. Yusuf S, Zucker D, Peduzzi P, et al. Effect of coronary artery bypass graft surgery on survival: overview of 10 -year results from randomised trials by the 
Coronary Artery Bypass Graft Surgery Trialists Collaboration. Lancet 1994; 344: 563-70

8. Varnauskas E. Survival, myocardial infarction, and employment status in a prospective randomized study of coronary bypass surgery. Circulation 1985; 72(Pt 2): V90-101.

9. Rogers WJ, Coggin CJ, Gersh BJ, et al. Ten-year follow-up of quality of life in patients randomized to receive medical therapy or coronary artery bypass graft surgery. The Coronary Artery Surgery Study (CASS). Circulation 1990; 82: 1859-62.

10. The VA Coronary Artery Bypass Surgery Cooperative Study Group. Eighteenyear follow-up in the Veterans Affair Cooperative Study of Coronary Artery Bypass Surgery for stable angina. Circulation 1992; 86: 121-30.

11. Grüntzig AR, Senning A, Siegenthaler WE. Nonoperative dilatation of coronary-artery stenosis: percutaneous Transluminal Coronary Angioplasty. N Engl J Med 1979; 301: 61-8.

12. Henderson RA, Pocock SJ, Sharp SJ, et al. Long-term results of RITA-1 trial: clinical and cost comparisons of coronary angioplasty and coronary-artery bypass grafting. Lancet 1998; 352: 1419-25.

13. Rodriguez A, Mele E, Peyregne E, et al. Three-year follow-up of the Argentine Randomized Trial of Percutaneous Transluminal Coronary Angioplasty versus Coronary Artery Bypass Surgery in Multivessel Disease (ERACI). J Am Coll Cardiol 1996; 27: 1178-84

14. HammCW, Reimers J, IschingerT, RupprechtHJ, BergerJ, BleifeldW. A randomized study of coronary angioplasty compared with bypass surgery in patients with symptomatic multivessel coronary disease. N Engl J Med 1994; 331: 1037-43.

15. King SPIII, Lembo NJ, Weintraub WS, et al. A randomized trial comparing coronary angioplasty with coronary bypass surgery. N Engl J Med 1994; 331: 1044-50.

16. Feit F, Brooks MM, Sopko G, et al. Long-term clinical outcome in the Bypass Angioplasty Revascularization Investigation Registry: comparison with the randomized trial. Circulation 2000; 101: 2795-802.

17. The BARI Investigators. Seven-year outcome in the Bypass Angioplasty Revascularization Investigation (BARI) by treatment and diabetic status. J Am Coll Cardiol 2000; 35: 1122-9.

18. Brooks MM, Jone RH, Bach RG, et al. Predictors of mortality and mortality from cardiac causes in the Bypass Angioplasty Revascularization Investigation (BARI) randomized trial and registry. Circulation 2000; 101: 2682-9.

19. Detre KM, Guo P, Holubkov R, et al. Coronary revascularization in diabetic patients: a comparison of the randomized and observational components of the Bypass Angioplasty Revascularization Investigation (BARI). Circulation 1999; 99: 633-40.

20. Weintraub WS, Stein B, Kosinski A, et al. Outcome of coronary bypass surgery versus coronary angioplasty in diabetic patients with multivessel coronary artery disease. J Am Coll Cardiol 1998; 31: 10-9.

21. Mathew V, Garrat KN, Holmes DR Jr. Clinical outcome after multivessel coronary stent implantation. Am Heart J 1999; 138: 1105-10.

22. Moussa I, Reimers B, Moses J, et al. Long-term angiographic and clinical outcome of patients undergoing multivessel coronary stenting. Circulation 1997; 96: 3873-9.

23. Kornowski R, Mehran R, Satler LF, et al. Procedural results and late clinical outcomes following multivessel coronary stenting. J Am Coll Cardiol 1999; 33: 420-6.

24. Hernández-Antolin RA, Alfonso F, Goicolea J, et al. Results ( $>6$ Months) of stenting of $>1$ major coronary artery in multivessel coronary artery disease. Am J Cardiol 1999; 84: 147-51.

25. Rodriguez A, Bernardi V, Navia J, et al. Argentine Randomized Study Coronary Angioplasty with Stents versus Coronary Bypass Surgery in Multiple Vessel Disease (ERACI II): one year follow up results. J Am Coll Cardiol 2000; 35(sppl A) 8A.

26. Rodriguez A, Bernardi V, Baldi J, et al. Cost effectiveness analysis between percutaneous coronary interventions versus coronary bypass surgery in multiple vessel disease: long-term follow up results of the Argentine Randomized Study (ERACI II). J Am Coll Cardiol 2000; 35(sppl A):3A.
27. Rodriguez A, Bernardi V, Navia J, et al. Argentine Randomized Study: Coronary Angioplasty with Stenting versus Coronary Bypass Surgery in patients with multiple-vessel disease (ERACI II): 30-day and one-year follow-up results. J Am Coll Cardiol 2001; 37: 51-8.

28. Serruys PW, Van Hout BA, Van den Brand MJB, et al. The ARTS (Arterial Revascularization Therapies Study): background, goals and methods. Int J Cardiovasc Interv 1999; 2: 41-50.

29. Serruys PW, Costa MA, Betriu A, et al. The influence of diabetes mellitus on clinical outcome following multivessel stenting or CABG in the ARTS Trial. Circulation 1999; 100(suppl I): I-364.

30. Pereira CF, Bernardi V, Martinez J, et al. Diabetic patients with multivessel disease treated with percutaneous coronary revascularization had similar outcome than those treated with surgery: one year follow up results from two Argentine Randomized Studies (ERACI-ERACI II). J Am Coll Cardiol 2000; 35(suppl A): 3A.

31. Freed M, O`Neill W, Safian RD. Single-vessel \& Multivessel Angioplasty. In: Freed M; Grines C; Safian RD. The New Manual of Interventional Cardiology. Birmingham, Michigan: Physicians Press, 1998: 75-95.

32. Pocock SJ, Henderson RA, Rickards AF, et al. Meta-analysis of randomised trials comparing coronary angioplasty with bypass surgery. Lancet 1995; 346: 1184-9.

33. Serruys PW, De Jaegere P, Kiemeneij F, et al. A comparison of balloon-expandable-stent implantation with balloon angioplasty in patients with coronary artery disease. Benestent Study Group. N Engl J Med 1994; 331: 489-95.

34. Serruys PW, van Hout B, Bonnier H, et al. Randomised comparison of implantation of heparin-coated stents with balloon angioplasty in selected patients with coronary artery disease (Benestent II). Lancet 1998; 352: 673-81.

35. Fischman D, Leon MB, Baim DS, et al. A randomized comparison of coronarystent placement and balloon angioplasty in the treatment of coronary artery disease. Stent Restenosis Study Investigators. N Engl J Med 1994; 331: 496-501.

36. O`Neill WW. Multivessel balloon angioplasty should be abandoned in diabetic patients! J Am Coll Cardiol 1998; 31: 20-2.

37. Lincoff AM, Califf RM, Moliterno DJ, et al. Complementary clinical benefits of coronary-artery stenting and blockade of platelet glycoprotein IIb/IIIa receptors. N Engl J Med 1999; 341: 319-27.

38. Sousa JE, Costa MA, Abizaid A, et al. Lack of neointimal proliferation after implantation of sirolimus-coated stents in human coronary arteries: a quantitative coronary angiography and three-dimensional intravascular ultrasound study. Circulation 2001; 103: 192-5.

39. Waksman R, White L, Chan RC, et al. Intracoronary g-radiation therapy after angioplasty inhibits recurrence in patients with in-stent restenosis. Circulation 2000; 101: 2165-71.

40. Leon MB, Teirstein PS, Moses JW, et al. Localized intracoronary gamma-radiation therapy to inhibit the recurrence of restenosis after stenting. N Engl J Med 2001; 344: 250-6.

41. Raizner AE, Oesterle SN, Waksman R, et al. Inhibition of restenosis with b-emitting radiotherapy. Report of the Proliferation Reduction With Vascular Energy Trial (PREVENT). Circulation 2000; 102: 951-8.

42. Waksman R, Bhargava B, White L, et al. Intracoronary b-radiation therapy inhibits recurrence of in-stent restenosis. Circulation 2000; 101: 1895-8.

43. Teirstein PS, Massullo V, Jani S, et al. Catheter-based radiotherapy to inhibit restenosis after coronary stenting. N Engl J Med 1997; 336: 1697-703.

44. Teirstein PS, Massullo V, Jani S, et al. Three-year clinical and angiographic follow-up after intracoronary radiation. Results of a randomized clinical trial. Circulation 2000; 101: 360-5

45. Souza JE, Casta MA, Abizaid AC, et al. Sustained suppression of neointimal proliferation by sirolimus - eluting stents: one-year angiographic and intravascular ultrasound follow-up. Circulation, 2001; 104: 2007-11. 